\title{
Las alianzas Estado / empresas para la mejora de la educación en Argentina a principios del siglo XXI: de la filantropía a la incidencia sobre la política educacional
}

\author{
As alianças Estado / Empresas para melhoria da educação na Argentina em \\ princípios do século XXI: da filantropia ao impacto na política educacional \\ State / Corporate Foundations Partnerships in Education at the beginning of the \\ XXI Century in Argentina: From Philanthropy to Policy Influence
}

LAURA ROBERTA RODRÍGUEZ

\begin{abstract}
Resumen: Se presentan avances de una investigación cuyo objeto es identificar y caracterizar la forma y contenido de las alianzas público-privadas en educación, los cambios en las fronteras entre lo público y lo privado en la política educativa, y sus consecuencias para la democratización de la educación pública, en Argentina, en las décadas recientes. Con un abordaje cualitativo, y a través de búsquedas en Internet y análisis de fuentes documentales, se reconstruyeron las transformaciones más recientes de los discursos, actividades y alianzas con distintos niveles del Estado argentino en el campo de la educación de dos casos de Fundaciones Empresarias: Arcor y Bunge \& Born. Se relacionan esas transformaciones con los cambios en las capacidades estatales, fenómenos de hibridación de las instituciones educativas, y con transformaciones generales del capitalismo, en particular los modos en que se resuelve la contradicción entre capital y trabajo.
\end{abstract}

Palabras clave: Alianzas público-privadas; Educación; Fundaciones Empresarias; Política educativa.

Resumo: O artigo apresenta o progresso de uma pesquisa cujo objetivo é identificar e caracterizar a forma e o conteúdo das alianças público-privadas na educação, as mudanças nos limites entre o público e o privado na política de educação, e suas consequências para a democratização da educação pública na Argentina nas décadas recentes. Com uma abordagem qualitativa, e através de buscas na Internet e análise de fontes documentais, as transformações mais recentes de discursos, atividades e alianças com diferentes níveis do Estado argentino no campo da educação foram reconstruídas em dois casos de Fundações Empresariais: Arcor e Bunge \& Born. Essas transformações estão relacionadas a mudanças nas capacidades do Estado, aos fenômenos de hibridização das instituições educativas, além das transformações gerais do capitalismo, particularmente as formas pelas quais a contradição entre capital e trabalho é resolvida.

Palavras-chave: Alianças público-privadas; Educação; Fundações empresariais; Política educativa. 


\begin{abstract}
This paper advances some findings of ongoing research focused on public-private partnerships in education in Argentina, the changes in publicprivate boundaries in educational policies, and the consequences for public education democratization in recent decades. Using a qualitative approach, by means of extensive searches on Internet and analysis of documentary sources, we describe activities, discourses, projects and partnerships with different state levels, of two domestic corporate foundations: Arcor and Bunge \& Born. We relate those changes to other major changes in three areas: state capacities, processes of educational hybridization, and ongoing general transformations of capitalism, particularly the ways in which the conflict between capital and labor is solved.
\end{abstract}

Keywords: Public-Private Partnerships; Education; Corporate Foundations; Educational Policy.

\title{
LAS ALIANZAS ENTRE EL ESTADO Y LAS EMPRESAS EN EL CAMPO DE LA EDUCACION EN ARGENTINA. UNA PROPUESTA DE INVESTIGACIÓN
}

El trabajo comunica los avances de una investigación, cuyo objeto es identificar y caracterizar, en Argentina, la forma y contenido de las alianzas público-privadas en educación, los cambios en las fronteras entre lo público y lo privado en la política educativa, y sus consecuencias para la democratización de la educación pública en las décadas recientes. En el espacio heterogéneo de agentes definidos como "Tercer Sector", la investigación se centra en las alianzas entre el Estado y las empresas privadas que desarrollan acciones de filantropía y Fundaciones de origen empresario, impulsadas por organizaciones que, con su acción pedagógica y financiamiento, contribuyeron a la incorporación de modelos de elaboración y gestión de políticas, organización social y valores culturales que las presentan como formas necesarias y beneficiosas para la elaboración e implementación de políticas públicas, asociadas a concepciones de "empresa social", "capital social", "responsabilidad social corporativa" y "sustentabilidad empresaria". Organismos de financiamiento (Banco Mundial, BID) o agencias internacionales (UNESCO, OEA, OECD), think tanks y grupos académicos, promovieron estas concepciones y políticas con diferentes matices y propuestas (Luna \& Serrano, 1995) (Luna, 1997) (Gallup - UdeSa, 1998) (Austin, Herrero, \& Reficco, 2004) (Austin J. E., 2000) (Licha, 2002) (SEKN, 2005 y 2006) (GDFE, 2009) (Leidi et al, 2012) (Pinkasz, 2012) (SITEAL-SIPI, 2013) (Langan, 2016).

El relevamiento realizado para conocer el estado del arte muestra que el número y variedad de producciones sobre el tema se incrementó notablemente a partir de los 90. En general, las investigaciones relevadas se fundan en supuestos teóricos conceptualmente solidarios con los que se impulsa y fundamenta la 
actividad empresarial en educación, asumen las transformaciones en el papel del Estado como un fenómeno dado y, en varios casos analizan las experiencias como ejemplos de políticas sociales necesarias para el alivio de la pobreza y la exclusión social y educativa, con énfasis en estudios de programas, proyectos y acciones a nivel micro, la recuperación de las experiencias vividas por los sujetos y las comunidades involucradas y/o la evaluación de su impacto en términos del logro de los objetivos propuestos. En muchos casos se trata de relevamientos e investigaciones realizadas por los propios actores del Tercer Sector comprometidos en las alianzas. A diferencia de esos trabajos, la investigación en curso pone el foco en las transformaciones de las formas de intervención del Estado, de la elaboración, implementación y evaluación de políticas educacionales, como parte de cambios más generales en la actual coyuntura de la acumulación capitalista (Franklin, Bloch, \& Popkewitz, 2003) (Kamat, 2004) (Vinokur, 2004) (Brown, 2008) (Srivastaba \& Oh, 2010) (Daly, 2012) (Roelofs, 2015) (Lambelet, 2017). Se pregunta por las condiciones, los condicionantes y las posibilidades que esas alianzas tienen en Argentina para contribuir a la democratización educativa, concebida como el grado en que las intervenciones ayudan a materializar una experiencia educativa, social e individualmente significativa y cualitativamente semejante para todas las personas (estudiantes, docentes, familias), y permiten la apropiación, la creación y la crítica colectiva del conocimiento y de las condiciones en que estas actividades se desarrollan, sobre la base de una autodeterminación creciente. Se parte del supuesto de que esa contribución no está determinada únicamente por las bondades del diseño o de la fundamentación de estas asociaciones a partir de principios de participación, comunidad y derechos ciudadanos, sino por los resultados -siempre provisorios- de la lucha por hacer realidad un proyecto de democratización de la sociedad -incluyendo la definición misma de sus términos ${ }^{1}$.

Con un abordaje cualitativo, se reconstruyó el espacio social de acciones y discursos vigentes de estos agentes colectivos, cartografiando sus actividades y alianzas, entre sí y con diversos niveles del Estado argentino en el campo de la educación, a partir de búsquedas en Internet (Ball, 2008) (Ball, 2011) (Aaronson \& Reeves, 2002) (Haslam, 2004). Simultáneamente, se recolectaron y analizaron fuentes documentales originadas en esas empresas y fundaciones (materiales de difusión, notas periodísticas, documentos oficiales de las empresas y fundaciones, materiales elaborados en el marco de programas y proyectos derivados de las alianzas, materiales audiovisuales, etc.), con la finalidad de elaborar una descripción 
detallada del contenido de las alianzas que involucraban a distintos niveles del Estado.Sobre esta base, se seleccionaron dos casos cuyas características resultaron pertinentes para los objetivos de la investigación.

El artículo presenta entonces el avance realizado en la descripción general de dos casos seleccionados, cuyo estudio será profundizado en una segunda fase de la investigación. Se trata de acciones desarrolladas por dos Fundaciones de grandes empresas de origen nacional, creadas en momentos históricos diferentes (1960 y 1990), con distintas trayectorias en el campo de la filantropía y con programas consolidados que implican alianzas con el Estado Nacional, Estados Provinciales y Municipales en el campo de la educación pública obligatoria de niños/as y jóvenes de sectores desfavorecidos. El primero de los casos es el llamado "Programa Sembrador", cuyo origen se remonta a 1974, desarrollado por la Fundación Bunge y Born. Durante algo más de treinta años el Programa se limitó a la donación de útiles escolares, hasta que en 2008 se amplió para incorporar la oferta de cursos a distancia para maestros (enseñanza de la lecto-escritura, ciencias naturales, matemática y lengua) becas para cursos presenciales sobre gestión escolar dirigidos a directores y supervisores, y acciones de acompañamiento a escuelas técnicas rurales para la Certificación ISO9001 (esto último, a partir de 2006). Estas acciones se desarrollan legitimadas por convenios, cartas de intención o auspicios de los ministerios de educación nacional y provinciales (Córdoba, Buenos Aires), conformando una verdadera alianza público-privada.

La otra experiencia es la del denominado "EduCómetro", apoyada por la Fundación Arcor (fundación de una empresa industrial de alimentos con proyección regional), y producto de una alianza con una organización privada, el CEADEL, (Centro de Apoyo al Desarrollo Local, fundado en 1986 por "expertos" en la problemática de la pobreza, el desarrollo local, la infancia y adolescencia, vinculados a UNICEF, ILPES /Chile y CIDES/ OEA)². Desde 2009, Fundación Arcor promueve la aplicación del Educómetro como herramienta diagnóstica para relevar y valorar las "oportunidades educativas comunitarias" en el nivel municipal, con la finalidad de diseñar intervenciones en pro del derecho a la educación de niños y jóvenes. La aplicación implica alianzas con más de diez Municipios en las provincias de Córdoba, Catamarca, Buenos Aires y Tucumán,

2

Entre 1998 y 2001 CEADEL recibió financiamiento de la Kellog Foundation para relevar "experiencias exitosas" de ONGs en el campo del desarrollo social e identificar "estilos de liderazgo organizacional" a replicar y fortalecer. En 1999 firmó un convenio con el PROAME (Programa de Atención a Niños y Adolescentes en Riesgo) del Ministerio de Desarrollo Social Nacional y financiamiento del BID, para realizar un diagnóstico de las capacidades institucionales de organizaciones que en 5 provincias argentinas desarrollaban actividades educativas y de promoción social. 
y el apoyo de otras fundaciones empresarias con actividad en la respectiva región de influencia. El trabajo describe sintéticamente ambos casos, y concluye con la presentación y discusión de hipótesis provisionales.

\section{LA FUNDACIÓN BUNGE \& BORN Y EL PROGRAMA "SEMBRADOR"}

La Fundación Bunge y Born fue creada en 1963 por los dueños de la empresa homónima, fundada a fines del siglo XIX en Argentina por comerciantes de origen holandés para comercializar y exportar granos y oleaginosas ${ }^{3}$. Nacida en la fase de expansión y diversificación de la compañía en contextos de políticas regionales desarrollistas, su objetivo fue "fomentar y estimular la investigación científica" a través de un premio al trabajo científico en diversas especialidades, al que se agregaron diversos premios en las décadas siguientes. En 1974 la Fundación inició el Programa de Ayuda a Escuelas Rurales (primarias), basado en la donación de útiles escolares y materiales educativos para niños, maestros e instituciones. Inicialmente abarcó a 40 escuelas, llegando a 800 en 1984. En 1994, en virtud de un convenio con el Ministerio de Educación Nacional, llegó con sus donaciones, a 1250 escuelas. Fue renombrado "Programa Sembrador", como un componente del Programa de Educación Rural, que en 1999 anudó una alianza estratégica con la Fundación Pérez Companc para compartir el financiamiento en partes iguales, manteniendo la Fundación B\&B la responsabilidad de la gestión operativa (coordinación, supervisión y evaluación), previa conformidad -renovada anualmente- de la Fundación Pérez Companc. Otras Fundaciones fueron sumándose paulatinamente ${ }^{4}$. En el año 2000, la Fundación B\&B incursiona en temas de capacitación financiando el Programa "La enseñanza de la lengua y la matemática en contextos de diversidad" en tres institutos de formación docente de la Provincia de Buenos Aires (San Cayetano, González Chaves y Tres Arroyos),

\footnotetext{
3 La empresa fue creada por un grupo inversor argentino de la industria agropecuaria / alimentaria, con base en la tradicional empresa "Molinos Rio de la Plata”.Se expandió a otros países y diversificó sus operaciones incorporando rubros de la industria alimentaria y de servicios a lo largo del siglo XX, reestructurándose y vendiendo activos para concentrarse nuevamente en la comercialización, acopio y exportación de granos, procesamiento de oleaginosas, producción y venta de fertilizantes, bioenergía y elaboración de productos finales para alimentación animal y humana, ahora como Bunge Cono Sur. Según su Reporte de Sustentabilidad 2016, tiene alrededor de 2000 empleados (de los cuales sólo el 57\% se enmarcan en convenios colectivos de trabajo), ventas netas por u\$s 3.945 millones, e inversiones sociales en la región por u\$s 976.338,12 de las que el 75\% se aplicaron a proyectos educativos. El 97\% de ese monto corresponde a inversiones en Argentina, y el resto en Uruguay, Paraguay y Bolivia.

4 Desde 1997, Fundación B\&B trabaja junto con la Fundación Navarro Viola en el desarrollo del Programa en escuelas rurales de las provincias del Chaco y Misiones, inicialmente a través de las Fundaciones Antorchas y Escolares, desde 2008 en forma directa. También participan del Programa otras Fundaciones, como Arcor, YPF y Banco Galicia.
} 
destinado a maestros y supervisores de escuelas rurales, con el aval de la Dirección General de Cultura y Educación de la Provincia. En 2008, el Programa fue objeto de una evaluación externa ${ }^{5}$ que determinó la ampliación de las líneas de trabajo y la redefinición de los objetivos, centrándose en el de "Colaborar en la mejora de la calidad educativa que ofrecen las escuelas rurales", alcanzando a algo más de 860 escuelas (Fundación Bunge y Born, 2014, pág. 24). Pasa, así, de una estrategia "asistencialista" a una estrategia "compensatoria" (Fundación Bunge y Born; Fundación Pérez Companc, 2015, pág. 43). Desde la concepción del programa, la escuela rural debía ser abordada en su inserción en el contexto social, económico, y hasta "climático o de infraestructura" ("existen tantas escuelas rurales como distintas realidades locales"), pero en todas, "el maestro rural juega un rol fundamental que se amplía y diversifica. Es educador, asistente social, gestionador y referente comunitario. Ante todo, el buen director es el que hace una buena escuela'. Se debía mejorar la concepción y la forma de la ayuda para que fuera sistemática, respondiera a las necesidades de la escuela y la involucrara en la acción, haciéndola sentirse "parte de un programa". (Pinkasz, Daniel (Coord.), 2012, pág. 62). Se decidió trabajar promoviendo una mayor participación de los destinatarios, proveyendo formación académica, solicitando para ello los avales correspondientes a los Ministerios de Educación provinciales, entendida como "una decisión estratégica" para "articular acciones público-privadas" (Pinkasz, Daniel (Coord.), 2012, pág. 52). Se organizaron, entre otras acciones, capacitaciones a distancia para maestros rurales, becas para cursos presenciales sobre gestión escolar destinados a directores y supervisores. Las nuevas líneas de trabajo se presentaban como "acciones especialmente pensadas para generar sinergia entre ellas así como nuevos modos de participación en el programa, en pos del mejoramiento de la calidad educativa. De esta manera, quienes realizan el curso presencial de gestión son los futuros líderes para llevar a cabo acciones conjuntas tales como cursos a distancia e implementación de proyectos de mejora en las escuelas", fomentando el vínculo entre los docentes y las fundaciones participantes (Pinkasz, Daniel (Coord.), 2012, pág. 52).

El programa de capacitación para maestros rurales -"que no pueden acceder a cursos presenciales"- comenzó en 2009 como Aula Virtual, compuesto por tres módulos: pedagógico (cursos de matemática, lengua, ciencias naturales), de gestión y cultural (nutrición), incluyendo la entrega de bibliografía y materiales didácticos. Sobre una base de convocatorias anuales el equipo de la Fundación $\mathrm{B} \& \mathrm{~B}$ selecciona provincias y, dentro de ellas, zonas de supervisión educativa. A institucional y publicitaria”), y GEA Research for Strategy, especializada en estudios de mercado. 
partir de allí, trabaja en alianza con el supervisor local, que se convierte en una figura central del Programa, en tanto participa de la selección de los cursos, forma parte del sistema de acompañamiento, seguimiento y evaluación, y es considerado factor clave en la mejora de los porcentajes de finalización

\begin{abstract}
"que ascendió de un 66\% inicial (año 2009) al 81.3\% (año 2011). Este vínculo se alimenta de distintas instancias de interacción: durante el curso reciben un reporte permanente del desempeño de los docentes provistos por las tutoras, se realizan encuestas de satisfacción a los maestros luego del presencial y al finalizar el curso y el supervisor elabora un informe que nos permite analizar en conjunto cómo seguir trabajando y qué propuestas hacer al equipo de docentes de su zona. En muchos casos, al finalizar el curso, el supervisor arma un plan de trabajo con propuestas de actividades". (Pinkasz, Daniel (Coord.), 2012, pág. 62)
\end{abstract}

Durante el curso, el equipo de la Fundación se hace cargo de las tutorías y consultas tanto de contenido, como de manejo técnico o de organización de los acompañamientos; incluye instancias presenciales con asistencia del capacitador, de personal de la Fundación a la zona, buscando "generar redes entre colegas y procurar el trabajo colaborativo" (Pinkasz, Daniel (Coord.), 2012, pág. 62). En 2010 comenzó un Programa de Capacitación de verano, "Herramientas de Gestión para Directores y Supervisores en Contextos Rurales" (alcanzando a 40 funcionarios por año). Paralelamente, en 2009 se realizó la primera convocatoria del concurso Proyecto Alfredo Hirsch, para elegir escuelas secundarias agrotécnicas con los mayores niveles de calidad y exigencia ${ }^{6}$. Esas escuelas conformaron la Red de Escuelas Alfredo Hirsch, dando continuidad a las capacitaciones, auditorías, evaluaciones de los alumnos y planes de mejora.

\title{
LA FUNDACIÓN ARCOR Y EL "EDUCÓMETRO”
}

Arcor es una industria del rubro alimentación, productora de golosinas, originada en 1951 en Arroyito (Córdoba) como empresa familiar, vinculada a

\footnotetext{
6 De 53 escuelas inscriptas, un jurado compuesto por figuras conocidas en el medio educativo como J.J.Llach y Alfredo van Gelderen, eligió a las doce mejores; las escuelas seleccionadas trabajaron con un equipo de capacitadores por un año y medio en temas de "calidad, ética y vocación docente, aprendizaje organizacional, el agro del futuro y niveles de empleo y desempleo juvenil”, y se contrató a la consultora noruega Det Norke Veritas para realizar auditorías y seleccionar a las cinco escuelas mejor calificadas, y certificar ISO 9001
} 
la familia Pagani ${ }^{7}$ En 1991 el Grupo creó la Fundación del mismo nombre; en 2004 fundó el Instituto Arcor para replicar la misión de la Fundación en Brasil, donde expandieron inversiones y emprendimientos productivos y comerciales; y finalmente, en 2015, la Fundación Arcor Chile. Según sus estatutos, su propósito es "realizar, promover y apoyar actividades e investigaciones sobre temas que contribuyan al progreso científico, cultural y al desarrollo económico e industrial de Argentina y Latinoamérica" y su misión "contribuir al progreso y bienestar de las personas, apoyando y promoviendo programas que, mediante la aplicación práctica del conocimiento y el uso eficiente de los recursos, satisfagan las necesidades locales de la comunidad" (Arroyo \& Estébanez, 1998, pág. 2 y ss.). Comenzó con acciones de apoyo a instituciones en el campo del arte (conciertos, seminarios, promoción de la expresión artística de niñas/os y jóvenes con capacidades especiales), el lanzamiento de un programa de padrinazgo a escuelas, y de fomento de la conciencia ecológica en docentes y alumnos -Educar Forestando- ${ }^{8}$.

Según Arroyo y Estébanez, que elaboraron un informe sobre el "modelo Arcor" de alianza público-privadas para el alivio de la pobreza -con financiamiento del Banco Mundial-, hacia 1996 la Fundación había abandonado el modelo "asistencialista" para adoptar otro "de promoción”; sus concepciones y métodos integraron la herramienta del concurso de proyectos, y la planificación y gestión profesional de las acciones con criterio de inversión antes que de donación (contratando un Gerente Técnico y un staff de profesionales), y se avanzó en la construcción de alianzas con distintos referentes del Estado, la "sociedad civil" y otras Fundaciones (Antorchas, Bunge y Born, Navarro Viola, entre otras) y organizaciones (UNICEF Argentina, IIPE/ UNESCO Buenos Aires), con foco exclusivo en la infancia y la adolescencia. En temáticas educativas se abrieron diversas líneas de acción: (a) Apoyo financiero y capacitación docente a Centros de Atención a la Discapacidad -Programa Superando Límites- de entidades sin

$7 \quad$ La empresa tuvo una rápida expansión, tanto en el mercado interno (con operaciones en todo el territorio nacional) como de exportación. En la década de los 70 amplió sus operaciones localizando unidades de producción en seis localidades de otras provincias argentinas, diversificando inversiones y abriendo filiales en Paraguay, Uruguay y Brasil. La ampliación continuó con nuevas adquisiciones en los 90, transformándose en un Grupo con presencia regional y oficinas en Canadá, México, Chile, Venezuela, Sudáfrica, China y España. En los 2000 el Grupo adquirió status de multinacional por su asociación con el Grupo Danone de Francia, el Grupo Bimbo de México, y en 2010 con Coca Cola. Según el Reporte de Sustentabilidad 2016 (ARCOR, 2017), tiene alrededor de 21.000 empleados (de los cuales el $20 \%$ se encuentra por fuera de convenios colectivos de trabajo), ventas por u\$s 2.800 millones, e inversiones sociales por $\$ 82$ millones. Participa en 70 organizaciones sociales y/o empresarias.

8 (FUNDACION ARCOR. Informe de Actividades 2006, p. 17.Recuperado el 18/10/2015 de http://www.fundacionarcor.org/es/detalle/127/informe-de-actividades-2006). 
fines de lugro que atienden a niños/as y jóvenes, en algunos casos articuladas "con otras acciones de nivel estatal para complementar los proyectos encarados" (Arroyo \& Estébanez, 1998, pág. 4); (b) Formación Humana y Ambiental, de apoyo a escuelas, instituciones sin fines de lucro que trabajan con adolescentes y jóvenes y áreas de cultura de Municipios de las provincias de Córdoba, Buenos Aires y otros centros urbanos, o financiando el Proyecto "Biblioteca a los cuatro vientos" para escuelas primarias (con Fundación Minetti); (c) Fondos Cooperativos con otras Fundaciones para financiar proyectos educativos: por ejemplo, con la Fundación ANTORCHAS para fortalecer el trabajo de ONGs que se dedican a juventud y minoridad (recuperación nutricional, prevención en comunidades de origen de "chicos de la calle", formación cultural infanto-juvenil en comunidades pobres, y prevención de discapacidad en esas mismas comunidades) a través del Programa Infancia y Desarrollo. Con la Inter-American Foundation estableció un Fondo (FEPIC, Formación, Educación, Prevención e Integración Comunitaria) para canalizar recursos a ONGs, organizaciones de base e instituciones sin fines de lucro que trabajan con población pobre hacia la atención primaria de la salud, capacitación y recreación, fundamentados y articulados con el concepto de "desarrollo de comunidades de base", e interesados en las "acciones colaborativas" de "evaluación y difusión de las experiencias y metodologías más exitosas, tratando de influir positivamente en las políticas generadas desde el sector público y/o de las agencias que se especializan en temas que afectan a la juventud" (Arroyo \& Estébanez, 1998, págs. 6-7). El informe concluía que el "modelo Arcor" se basaba en un conjunto de proyectos y tareas con varios "socios" según el objetivo que la empresa establecía para cada Programa, con preferencia organizaciones comunitarias y entidades sin fines de lucro antes que representantes de los diversos niveles del Estado.

Si bien el socio principal solía ser el mismo Arcor, la apertura de alianzas con otras Fundaciones donantes y la metodología de presentación de proyectos había abierto la posibilidad de consolidar el formato de financiamiento con contrapartes, y había llevado "a que en la práctica las relaciones con el Estado (de manera directa o indirecta por medio de las vinculaciones de las instituciones receptoras) se amplíe y establezca modelos de asociaciones parciales que parecen tender a expandirse en los próximos años” (Arroyo \& Estébanez, 1998, pág. 10). Sin embargo, el sector público como contraparte no era un objetivo prioritario a fines de los 90, probablemente porque las Fundaciones Empresarias sentían que "'hacen el trabajo que el Estado no hace' y tenían temor a quedar pegados [sic] políticamente al funcionario de turno" (Arroyo \& Estébanez, 1998, pág. 13). De todos modos, en la medida en que las organizaciones sociales con las que trabajaba participaban, a su vez, de programas sociales, el "entrecruzamiento" 
con el Estado era cada vez más habitual y generaba espacios de acción común, aún cuando no quedara "del todo claro cuál es la contraparte estatal" (Arroyo \& Estébanez, 1998, pág. 11).

Hacia 2005, el Grupo decidió enmarcar todas sus acciones en el modelo de Responsabilidad Social Empresaria (RSE), creando un Programa y una Gerencia de RSE. En 2007 aprobó la Política de Inversión Social Arcor (ISA), y en 2009 migró al concepto de "Sustentabilidad", elaborando su Estrategia y Plan de Sustentabilidad para los próximos años y definiendo una "Política de Sustentabilidad Arcor” (Kosakoff, 2014, pág. 94). En 2015 El Comité Corporativo de Sustentabilidad aprobó la "Política de Compromiso con los Derechos del Niño", siguiendo la Iniciativa Derechos del Niño y Principios Empresariales lanzada por el Pacto Global de ONU /UNICEF/Save the Children, convirtiéndose en un actor dinámico en la promoción de actividades de sensibilización de la opinión pública y de redes de organizaciones público-privadas en la temática.

Todos estos cambios impactaron en la actividad de la Fundación, cuya modalidad de trabajo pasó a estar centrada en alianzas sistemáticas y sinérgicas con actores locales, organizaciones de base, y comunidades, involucrándolas "desde el comienzo (diagnóstico), apropiándose de los proyectos y los espacios generados junto a las otras organizaciones" (Pinkasz, Daniel (Coord.), 2012, pág. 25), brindando "a las organizaciones comentarios y devoluciones a los proyectos antes de ser recibidos, como un acompañamiento antes de la recepción definitiva para su evaluación", capacitación en temáticas relativas a la "infancia (educación, vulneración de derechos, etc.) y en temas inherentes al fortalecimiento interinstitucional, gestión para la elaboración de informes programáticos y financieros, relevamientos, sistematización y registro de las actividades que realizan, búsqueda de recursos, etc." (Pinkasz, Daniel (Coord.), 2012, pág. 25). Dando cuenta de esta evolución, los documentos institucionales a la fecha de elaborar este artículo, definen la misión de la Fundación como "contribuir para que la educación se convierta en un instrumento de igualdad de oportunidades para todos los niños", con dos objetivos principales: "generar y compartir conocimientos y metodologías de trabajo sobre la inversión social, y contribuir a instalar en la sociedad y en la agenda pública la causa de la infancia para convocar, de esta manera, a que otros actores se sumen a ella", mediante cuatro líneas de acción: "Iniciativas Territoriales", "Capacitación y Formación", "Estudios e Investigación" (sola o asociada con centros de estudios y unidades académicas), y "Movilización social y pública" (de actores públicos y privados a través de estudios, seminarios, talleres y convenios con empresas y municipios) ${ }^{9}$. Se adoptó 
el enfoque de "Oportunidades Educativas Comunitarias" (OEC) a partir del modelo de "gestión social asociada" y el "diagnóstico participativo", entendidos como "un modo de planificación y gestión realizado en forma compartida entre OSC, organizaciones estatales y del sector privado", pensado para "incrementar y mejorar las oportunidades educativas locales, a través del fortalecimiento y la profundización de la democratización en la toma de decisiones compartidas, superando un modo fragmentado de funcionamiento entre Estado-SociedadSector privado" (Pinkasz, D. (Coord.), 2012, págs. 69-70). El Educómetro deriva de esta línea de acción, en virtud de una alianza con el CEADEL ${ }^{10}$. Se lo define como una herramienta para medir las "oportunidades educativas comunitarias" de niños, niñas y adolescentes en un territorio determinado, entendiéndolos como indicadores del grado de desarrollo y del capital social disponible; utiliza diversidad de instrumentos de recolección de datos cualitativos y cuantitativos: planillas pautadas, entrevistas, cuestionarios, talleres, estimaciones del gasto público, etc., para caracterizar cuatro dimensiones de una comunidad: condiciones de vida, infraestructura y capital social existente, situación del sistema educativo formal, oferta de servicios y espacios de educación no formal, y políticas, programas sociales locales y gasto público destinado a la infancia y la adolescencia.

A tono con la Estrategia 2020 para el Sector Educativo del Banco Mundial -apoyada en dos ideas-fuerza: la importancia del aprendizaje y no únicamente de la escolarización, y que el aprendizaje puede lograrse dentro y fuera de la escuela- (BIRF / BM, 2011), el Educómetro supone una concepción amplia de la educación, como el conjunto de "interacciones deliberadas o espontáneas en las que se pone en juego la transmisión de una cultura heredada" e involucra "la articulación de la escuela y la comunidad, y la relación entre éstas y las diversas alternativas de la educación no formal": lo educativo "está presente en las aulas pero, a la vez, atraviesa prácticas familiares, interacciones sociales, ofertas socioculturales, deportivas, recreativas y medios de comunicación". Vendría de este modo a articular y potenciar esas oportunidades, optimizando la gestión de las organizaciones de la sociedad civil y la elaboración de agendas de acción colectivas, a través de una mayor incidencia en las políticas públicas para la infancia (FUNDACIÓN ARCOR, 2012, págs. 2-3) (Cardarelli \& Nirenberg,

\footnotetext{
10 El CEADEL, (Centro de Apoyo al Desarrollo Local) se define como una ONG de carácter profesional, fundada en 1986 por expertos en pobreza, desarrollo local, infancia y adolescencia vinculados a UNICEF, ILPES Chile y CIDES/OEA. En su página web informa que su finalidad es “promover y apoyar técnicamente la gestión de las administraciones locales y provinciales, así como de las organizaciones comunitarias y de la sociedad civil, en torno a la formulación y gestión de las políticas sociales (programas/proyectos)”, y sus integrantes conforman "un grupo multidisciplinario de larga trayectoria en la función pública, así como en el campo de los programas sociales, la investigación-acción y la investigación aplicada a la solución de los problemas desde esferas no gubernamentales", siendo sus principales referentes Olga Nirenberg y Graciela Cardarelli. Ver: http://ceadel.org.ar/institucion.htm.
} 
2012). Educómetro se propone tanto "generar una instancia de discusión con el actor público", como promover la "construcción de organizaciones sociales" (FUNDACIÓN ARCOR, 2011, pág. 30), en un contexto local con capacidades infraestructurales debilitadas, tal como parecerían reflejar las declaraciones públicas de varios funcionarios municipales, notando "la falta" de herramientas, iniciativas o capacidades estatales locales para realizar diagnósticos y formular planes de acción.

En 2010 esta herramienta se aplicó como prueba piloto en dos municipios de dos provincias, y hasta el 2016 había alcanzado al menos a 23 municipios en ocho provincias, en muchos casos localidades en las que la empresa tiene sus plantas.. En 2014 se adaptó el Educómetro para que se constituyera en un dispositivo "autoaplicable"; se comenzó a estimular a los municipios a usar los diagnósticos para diseñar planes locales destinados a la infancia. Educómetro certificó las normas ISO 9001:2008 DNV-GL-RvA, y se efectivizó una alianza de colaboración con el Programa Nacional Mapa Educativo de la Subsecretaría de Planeamiento Educativo del Ministerio de Educación ${ }^{11}$.

\section{ALGUNAS HIPÓTESIS: CAPACIDADES ESTATALES, HIBRIDACIÓN Y CONTRADICCIONES ENTRE CAPITAL Y TRABAJO}

El análisis de las particularidades de los dos casos presentados permite adelantar algunas hipótesis sobre el sentido de estas transformaciones. En un nivel descriptivo, observamos una transformación de los objetivos, contenidos y forma de las acciones de empresas y fundaciones empresarias en temas de educación, que en Argentina parecería mostrar una creciente intervención en el corazón -y no solamente en la "periferia"- del sistema educativo. Esta transformación se condensa en la preeminencia que adquiere, en la agenda de la Responsabilidad Social Empresaria, el objetivo explícito de incidir en la política educativa Sin abandonar las formas de intervención tradicionales, generalmente individuales y de corte claramente filantrópico (donaciones en dinero o especie, financiamiento de becas y premios, desarrollo de acciones de educación no formal, oferta de cursos / charlas sobre temáticas relativas al núcleo de actividad de la empresa como parte de

11 Según el Coordinador del Programa, Daniel Sicotti, en 2014 esa alianza permitiría generalizar la aplicación del Educómetro en los más de 2000 municipios del país. Mapa Educativo se inició en 2003 como sistema de información geográfica para relevar, junto con los Ministerios de Educación provinciales, la distribución territorial de la oferta de educación pública y privada en todos los niveles, modalidades de la educación formal y no formal y tipos de gestión. Según Sticotti, el vínculo con Arcor surgió de la solicitud de información que realizó la Fundación cuando aplicaba el Educómetro en algunos municipios. Ver: www. mapaeducativo.edu.ar (Acceso el 3/10/2016). 
las estrategias de marketing, apoyo financiero a la edición de materiales educativos complementarios, entre otros), comienzan a tener cada vez más peso e interés acciones en red que abordan sistemáticamente asuntos "centrales" de la política sectorial, tales como el diagnóstico con fines de planificación, o los contenidos de la capacitación de funcionarios del sistema y de docentes, acompañadas por acciones de sensibilización de la opinión pública y la construcción de agenda. En las primeras décadas del 2000, no sólo se expanden las actividades de las Fundaciones empresarias, sino que muchas empresas crean, dentro de sus estructuras, áreas a cargo de proyectos de RSE, rápidamente integrados al paradigma difundido globalmente de la "Sustentabilidad Corporativa", aspirando a la "profesionalización, de programas medibles y organizados, de un plan con objetivos y resultados", y a hacerlo "en conjunto con organizaciones sociales, ministerios y gobiernos que operan en las comunidades que intervenimos". ${ }^{12}$

En un primer nivel de análisis, estas alianzas son una forma de intervención facilitada por el "debilitamiento" de las capacidades estatales; ello podría incrementar la permeabilidad y aceptación de las intervenciones por parte de los agentes de los niveles más bajos del Estado e incluso de funcionarios ministeriales de las distintas provincias, así como de la legitimidad, valoración positiva y naturalización por parte de docentes, directivos, supervisores, estudiantes y familias.

Avanzando un poco en el análisis para integrar estos cambios en procesos más generales del campo educativo, resulta sugerente la afirmación de V. Karpov y E. Lissovska (1998) ${ }^{13}$, quienes acuñaron el concepto de "hibridación de las instituciones educativas", para referirse específicamente a la mutación de las relaciones de poder que subyacen a una nueva división de responsabilidades, que dejan de ser controladas por "un centro"; además, construyen una nueva utopía educativa basada en un entramado de estructuras de decisión que dificulta "discernir quién decide" (Vinokur, 2004, pág. 8) [en itálica en el original]. En esas estructuras híbridas, el problema político de los fines de la educación y la distribución de los recursos parece quedar expurgado vía alianzas, convenios, etc., que los sustraen de la deliberación pública, pues la "buena gobernanza" conlleva la opacidad creciente de las decisiones y la transparencia creciente de la ejecución.

\footnotetext{
12 Cecilia Barbón Directora de la Fundación Acindar. TRES MANDAMIENTOS, “Las fundaciones empresarias en la era de la sustentabilidad", 5/07/2016. Recuperado el 8 de noviembre de 2016 de http://www. tresmandamientos.com.ar/nota/663-las-fundaciones-empresarias-en-la-era-de-la-sustentabilidad

13 Karpov V. et Lissovska E., 1998 - Untitled, Working paper, Western Michigan University. Los autores acuñan este concepto para analizar el proceso de borramiento de las fronteras entre lo público y lo privado en la educación de la Rusia post-soviética. Citado por Vinokur (2004).
} 
Si se atiende ahora a las transformaciones sociales más generales, corresponde analizar estas alianzas en el marco de la historia de las relaciones de fuerza políticas y del proyecto social predominante a escala nacional, en su articulación con la internacional (Jessop, 2003, 2015a, 2015b). En este sentido, es posible que en países que, como Argentina, lograron construir sistemas educativos públicos más o menos centralizados dentro de sociedades con aparatos de hegemonía privadas relativamente débiles, se haya producido en las décadas reciente una fase de conformación y fortalecimiento de organizaciones de la sociedad civil y de expansión de formas organizadas de filantropía empresaria, seguidamente potenciadas por los nuevos paradigmas de la RSE y la Sustentabilidad Corporativa (incremento de la cantidad de fundaciones, conformación de redes de fundaciones y empresas y su integración en la escala local e internacional). Ese proceso estaría facilitando el procesamiento de cambios profundos en las relaciones con el Estado y en sus formas de intervención en la cuestión social, expandiendo a escala global los "arreglos" socio-políticos característicos de la formación social estadounidense, en la que, para algunos autores, las fundaciones se han tornado el "brazo planificador" del sistema político, sustituyendo a las instituciones políticas -como por ejemplo los partidos programáticos- en el diseño de reformas y medidas de política (Arnove \& Pinede, 2007) (Roelofs, 2015).

Ahora bien, debe recordarse que la educación es uno de los espacios en los que se juega el conflicto entre trabajo y capital, y el conflicto entre grupos sociales. Como afirma A. Vinokur (2004), desde el punto de vista de los intereses del capital, la forma actual de ese conflicto debe superar dos obstáculos, el primero es la baja rentabilidad de las inversiones en esos sistemas (por la dificultad para generar economías de escala y ganancias de productividad); el segundo se relaciona con las funciones sociales de la instrucción y la educación, que constituyen un problema primariamente político. Gran parte de la literatura académica caracteriza estos cambios como un pasaje del gobierno a la gobernanza, la conformación de una "nueva capilaridad" en las fronteras entre lo público-estatal y lo privadosocial; para los enfoques pluralistas, las alianzas público-privadas son una potente fuente de innovación para resolver problemas sociales, que la acción convencional de los Estados de países periféricos parece actualmente ya no poder resolver. Desde otro marco de análisis, los enfoques críticos resaltan que las actividades del Tercer Sector funcionan como una capa protectora para el capitalismo, desviando la energía social de la concepción, promoción e implementación de alternativas que puedan cuestionar las bases profundas del sistema (Roelofs, 1995); permiten a la burguesía mantener el control de la definición de los problemas y de sus formas de resolución (Lambelet, 2017), produciendo una homogeneización de prácticas 
y concepciones entre otros agentes colectivos -organizaciones de la sociedad civil, organizaciones de base y organizaciones surgidas de los movimientos populares, etc.

Trabajando sobre estos ejes, se espera caracterizar la sustancia de esas alianzas, como ejemplo de la dinámica redefinición de las fronteras entre lo público y lo privado en educación, y su potencial para la democratización educativa.

\section{REFERÊNCIAS}

AARONSON, S.; REEVES, J. Corporate Social Responsibility in the Global Village: the Role of Public Policy. Washington D.C., NPA Report $N^{\circ}$ 36, National Policy Association 2002.

ARCOR. Reportes de Sustentabilidad. Varios años. Disponible en: http// www.arcor.com/downloads/es/. Acceso en: 20/08/2016.

ARCOR. Nuestra Historia. Disponible en http://www.arcor.com.ar/ downloads/es/Nuestra\%20Historia.pdf. Acceso en: 18/03/2016

ARNOVE, R.; PINEDE, N. Revisiting the "Big Three" Foundations. Critical Sociology, $\mathrm{N}^{\circ} 33$, p.389-425, 2007.

ARROYO, D.; ESTÊVANEZ, M.C. Programa Alianzas para la Reducción de la Pobreza. Estudio de Caso. Fundación Arcor. Buenos Aires, FLACSO / The World Bank Group, 1998.

AUSTIN, James E. The Collaboration Challenge. How Nonprofits and Businesses Succeed Through Strategic Alliances. San Francisco, Jossey-Bass Books, 2000.

AUSTIN, J.E.; HERRERO, G.; REFICCO, E. La nueva ruta: alianzas sociales estratégicas. Harvard Business Review América Latina, p. 1-10, 2004.

BALL, Stephen. New Philantropy, New Networks and New Governance in Education. Political Studies, vol..56, n.4, p.747-756, 2008.

BALL, Stephen. Política social y educativa, empresa social, hibridación y nuevas comunidades discursivas. Propuesta Educativa, vol. 20, n. 36, p.25-34, 2011. 
BERGER, G.; GOWLAND, C. La Fundación Arcor: Promoviendo Articulaciones Institucionales para el Desarrollo Social a Nivel Local. RedEAmérica. Casos Pedagógicos, p.6-32, 2007. Disponible en: http://www.redeamerica.org/ Portals/0/Publicaciones/Casos\%20Pedagógicos.pdf. Acceso en: 21 sept. 2015.

BIRF / BM. Aprendizaje para todos. Invertir en los conocimientos y las capacidades de las personas para fomentar el desarrollo. Estrategia de Educación 2020 del Grupo del Banco Mundial. Resumen Ejecutivo. Washington D.C., BIRF / BM, 2011.

BONEFELD, Werner. La razón corrosiva. Una crítica al Estado y al capital. Buenos Aires, Herramienta Ediciones, 2013.

BROWN, D. S. Who Gives, Who Receives, and Who Wins? Transforming Capital Into Political Change Through Nongovernmental Organizations. Comparative Political Studies, 41(1), p. 24-47, January 2008.

CARDARELli, G.; NIRENBERG, O. Método para el diagnóstico local de oportunidades educativas comunitarias. El Educómetro. Buenos Aires, CEADEL. Cuaderno N 49, 2012.

DALY, S. Philantropy as an Essentially Contested Concept. Voluntas, 23, p.535$557,2012$.

FRANKLIN, B.M.; BLOCH, M. N.; POPKEWITZ, T.S. (Edits). Educational Partnerships and the State: the Paradoxes of Governing schools. children and families. New York, Palgrave MacMillan, 2003.

FUNDACIÓN ARCOR. Informe de Actividades. Córdoba, Fundación Arcor, Varios Años.

FUNDACIÓN ARCOR. Educómetro. La infancia es la medida. Suplemento Especial. Tercer Sector, 88, 2015.

FUNDACIÓN BUNGE \& BORN. 1963-2013. Buenos Aires, Fundación Bunge \& Born, 2014. 
FUNDACIÓN BUNGE \& BORN - FUNDACIÓN PEREZ COMPANC. Las escuelas del medio rural. Avellaneda, Escuela Talleres Gráficos Manchita, 2009.

GRUPO DE FUNDACIONES Y EMPRESAS (GDFE). Estudio sobre Inversión Social Privada en Argentina 2008. Buenos Aires, GDFE, 2009.

HASLAM, P.A. The Corporate Social Responsibility System in Latin America and the Caribbean. Policy Paper PP 04 01- Ottawa, FOCAL (Fundación Canadiense para las Américas, 2004.

INSTITUTO GALLUP de ARGENTINA - UNIVERSIDAD de SAN ANDRÉS (UdeSA). Estudio de Filantropía Empresaria. Buenos Aires, Instituto Gallup de la Argentina - Universidad de San Andrés, 1998.

JESSOP, Bob. Governance and Metagovernance: On Reflexivity, Requisite Variety, and Requisite Irony. Lancaster University, Department of Sociology, 2003. Disponible en: http://www.comp.lancs.ac.uk/sociology/papers/JessopGovernance-and-Metagovernance.pdf. Acceso en: 14 ago.2010.

JESSOP, Bob. Political Capitalism, Economic and Political Crises, and Authoritarian Statism. Spectrum Journal of Global Studies [en línea], vol.7, n.1, 2015a. Disponible en: http://spectrumjournal.net/volume-7-issue-1-2015/. Acceso en: 14 mar. 2016.

JESSOP, Bob. The State: past, present, future. New York, Polity Press, 2015b.

KAMAT, S. The privatization of public interest; theorizing NGO discourse in a neoliberal era. Review of International Political Economy, Vol.11, $\mathrm{N}^{\circ} 1$, p.155-176, February 2004.

KOSAKOFF, B. Globalizar desde Latinoamérica: el caso Arcor (2001-2012). Del caramelo a los alimentos, de Arroyito al mundo. Boletín Informativo Techint, p. 69-106, mayo/agosto 2014.

LAMBELET, A. La philanthropie : usages du terme et enjeux de luttes. ethnographiques.org. Revue en ligne de sciences humaines et sociales [en línea], 2017. Disponible en: http://www.ethnographiques.org/2017/Lambelet. Acceso en 21 marzo 2017. 
LANGAN, C. La nueva inversión social: multiactoral, integral y colaborativa. EVOLUCION, vol. 5, n. 48, p.12-15, 2016.

LEIDI, P.; LANGLOIS, A.; ARANA SERNA, M.J. Diez años de RSE en Argentina. Hacia el camino de la sustentabilidad. Buenos Aires, ComunicaRSE, 2012.

LICHA, I. (ed.). Gerencia social en América Latina. Enfoques y experiencias innovadoras. Washington D.C., BID, 2002.

LUNA, E. F. Fundaciones Empresarias en el Campo Social en Argentina: Directorio, Mapa Institucional y Marco Legal y Tributario. Buenos Aires, GADIS - PNUD, 1997.

LUNA, E.F.; SERRANO, R. Luna, E., \& Serrano, R. La Filantropía Corporativa en la Argentina. Un Estudio Preliminar. Buenos Aires, GADIS, 1995.

OLMOS, L. E.; TORRES, C. A. (2009). Theories of The State, Educational Expansion, Development, and Globalizations: Marxian and Critical Approaches. En R. Cowen, \& A. Kazamias (Edits.), International Handbook of Comparative Education. Vol. 22. London, Springer, p. 73-86, 2009.

PINKASZ, Daniel (Coord.). Guía de Inversión Social Privada en Educación. Buenos Aires, GDFE - Escuela Talleres Gráficos Manchita, 2012.

ROELOFS, Joan. The Third Sector as a Protective Layer for Capitalism. Monthly Review, Vol. 47, N 4, p.15-25, September 1995.

ROELOFS, Joan. How Foundations Exercise Power. American Journal of Economics and Sociology, Vol. 74, N 4, p. 654-674, September 2015.

SITEAL - SIPI. La Responsabilidad Social Empresaria en el paradigma de los derechos del niño. Diálogos del SIPI. Conversación con Javier Rodríguez. IIPE / UNESCO. Buenos Aires, UNICEF - Fundación Arcor, 2013

SEKN (Social Enterprise Knowledge Network). Alianzas sociales en América Latina. Enseñanzas extraídas de colaboraciones entre el sector privado y organizaciones de la sociedad civil. New York, BID, 2005. 
SEKN (Social Enterprise Knowledge Network). Gestión efectiva de emprendimientos sociales. Lecciones extraídas de empresas y organizaciones de la sociedad civil en Iberoamérica. Washignton D.C., BID - David Rockefeller Center for Latin American Studies- Harvard University Editorial Planeta, 2006.

SRIVASTABA, P.; OH, S. A. Private foundations, philantropy, and partnership in education and development; mapping the terrain. International Journal of Educational Development, N³0, p. 460-471, 2010.

VINOKUR, Annie. Public, privé....o hybride? L'effacement des frontières dans l'éducation. Cahiers de la recherche sur l'éducation et les savoirs, $\mathrm{N}^{\circ} 3$, p. 13-33, 2004.

LAURA ROBERTA RODRÍGUEZ Magister en Política y Gestión de la Educación (UNLu), Doctoranda en Ciencias Sociales (UBA). Vinculada a Departamento de Educación - Universidad Nacional de Luján, Argentina.

Recebido em setembro de 2018 Aprovado em fevereiro de 2019 